Research Paper

\title{
Gambogenic Acid Exerts Antitumor Activity in Hypoxic Multiple Myeloma Cells by Regulation of miR-21
}

\author{
Ping Liu, Xue Wu, Lu Dai, Zheng Ge, Chong Gao, Hongming Zhang, Fei Wang, Xiaoping Zhang ${ }^{\bowtie}$, Baoan \\ Chen ${ }^{凶}$ \\ Department of Hematology and Oncology (Key Department of Jiangsu Medicine), Zhongda Hospital, Medical School of Southeast University, Nanjing 210009, \\ People's Republic of China. \\ $\square$ Corresponding authors: Xiaoping Zhang, Department of Hematology and Oncology (Key Department of Jiangsu Medicine), Zhongda Hospital, Medical \\ School of Southeast University, Nanjing 210009, People's Republic of China Tel +86 2583262473 E-mail violetzxp_32@sina.com Baoan Chen, Department of \\ Hematology and Oncology (Key Department of Jiangsu Medicine), Zhongda Hospital, Medical School of Southeast University, Nanjing 210009, People's \\ Republic of China Tel +86 2583272006 Fax +86 2583272011 E-mail cba8888@hotmail.com \\ (c) Ivyspring International Publisher. This is an open access article distributed under the terms of the Creative Commons Attribution (CC BY-NC) license \\ (https:// creativecommons.org/licenses/by-nc/4.0/). See http://ivyspring.com/terms for full terms and conditions.
}

Received: 2017.01.22; Accepted: 2017.08.31; Published: 2017.09.16

\begin{abstract}
Hypoxia is an inseparable component of the bone marrow (BM) microenvironment, accounting for aggressive tumor behavior and poor prognosis of multiple myeloma (MM). Gambogenic acid (GNA) has proven to be an attractive option for treatment of tumors due to its tumor suppressive activity. Herein, we found that GNA exhibits remarkable apoptotic activity against MM cells even under hypoxia. MicroRNA-21 (miR-21) has been found over-expressed in MM patients and associated with the occurrence and development of MM. Direct studies have shown that there is a functional link between hypoxia and miR-21 expression in multiple types of tumors. In the current study, we found that hypoxia increased miR-21 expression in U266 cells and miR-21 induced by hypoxia was associated with concurrent reductions in its target PTEN. After treatment with GNA, miR-21 expression in hypoxic U266 cells was strikingly downregulated in a dose-dependent manner. Besides, we identified that regulation of miR-21/PTEN by GNA under hypoxia is related with inhibition of HIF-l $\alpha$ accumulation and STAT3 phosphorylation. Furthermore, in vivo study revealed that intravenous GNA injection could significantly suppress tumor growth and the miR-21/PTEN pathway is involved in GNA's anti-tumor effects. Taken together, all these results indicated that GNA could be a highly potent therapeutic for human MM.
\end{abstract}

Key words: Multiple myeloma, bone marrow, Gambogenic acid

\section{Introduction}

Multiple myeloma $(\mathrm{MM})$ is one of the most common hematologic malignancy, characterized by accumulation of aberrant malignant plasma cells in the bone marrow (BM) microenvironment [1]. Introduction of novel therapeutics such as proteasome inhibitor bortezomib have greatly improved overall survival in MM patients [2,3]. However, high rates of relapse and resistance to drug therapies remains inevitable. Although the mechanisms remain unclear, adaptive responses induced by hypoxic conditions in the BM have been implicated to cause myeloma cells to become anti-apoptotic, glycolytic, and immature [4].
MicroRNAs (miRs) constitute a novel class of non-coding RNAs that act as negative modulators of gene expression [5]. miRs are involved in mediating various critical cellular processes such as proliferation, apoptosis, and differentiation [6, 7]. It was recently shown that hypoxia alters the expression of various miRs, which may also contribute to hypoxic adaptation [8, 9]. Aberrant expression of miR-21 within $\mathrm{MM}$ has been shown strongly correlated with disease progression, as well as being predictive of poor patient prognosis [10, 11]. Its expression is not only closely relevant to the tumor development, but also to the hypoxic environment. 
Thus, agents targeting the miR-21 may represent a potential therapeutic strategy to be used on hypoxic cells that survive other anti-tumor treatment.

In recent decades, agents derived from herbs have been recognized as novel cancer therapeutics. Gambogenic acid (GNA, Figure 1A) is one of the main components of gamboge, a traditional medicine exuded from the Garcinia hanburyi tree [12]. Previous studies suggested that GNA exhibits stronger and broader anti-tumor effects but lower toxicity than gambogic acid (GA), another component of gamboge which has been clinically applied to treat multiple cancers [13]. It has been reported that GNA can induce apoptosis by inactivation of various signaling pathways in human tumors [14-16]. Our previous study has revealed that GNA is cytotoxic toward MM cells under normoxic conditions [17]. Here in this study we assessed the anti-tumor activity of GNA in hypoxic MM cells and investigated whether miR-21 conferred an involvement in GNA-induced apoptosis under hypoxic conditions.

\section{Materials and Methods}

\section{Cell culture and establishment of hypoxic model}

The multiple myeloma cell U266, obtained from Shanghai Cell Bank of Chinese Academy of Sciences (Shanghai, China), were grown in RPMI-1640 medium (Gibco, Grand Island, NY, USA) at 15\% inactivated fetal bovine serum (Sijiqing, Hangzhou, China) with $100 \mathrm{U} / \mathrm{mL}$ penicillin and $100 \mu \mathrm{g} / \mathrm{mL}$ streptomycin (Sigma-Aldrich, St. Louis, MO, USA) at $37^{\circ} \mathrm{C}$ in a humidified atmosphere with $5 \% \mathrm{CO}_{2}$. For in vitro hypoxia conditions, cells were maintained in a sealed chamber flushed with a gas mixture $\left(94 \% \mathrm{~N}_{2}\right.$, $5 \% \mathrm{CO}_{2}$ and $1 \% \mathrm{O}_{2}$ ).
A

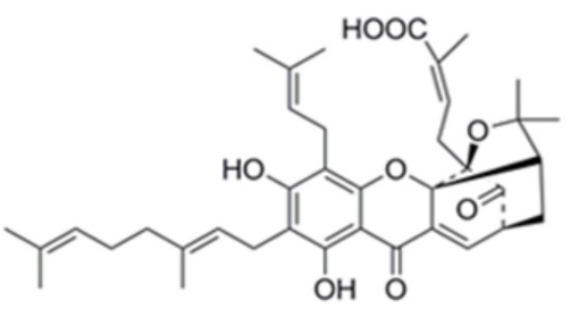

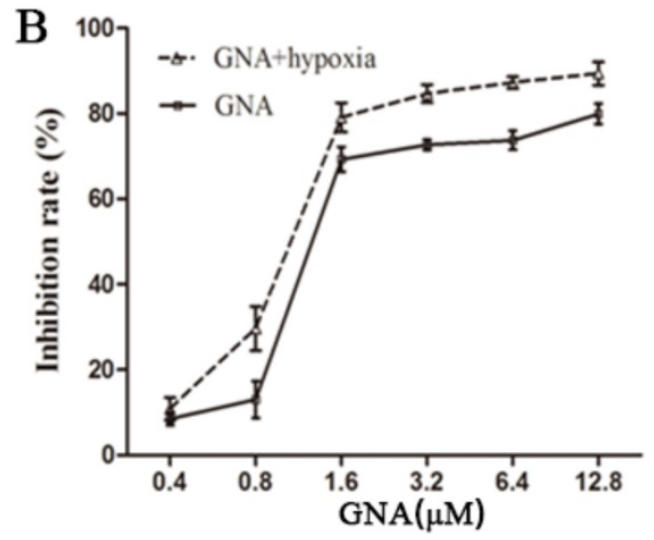

$0.8 \mu \mathrm{M}$
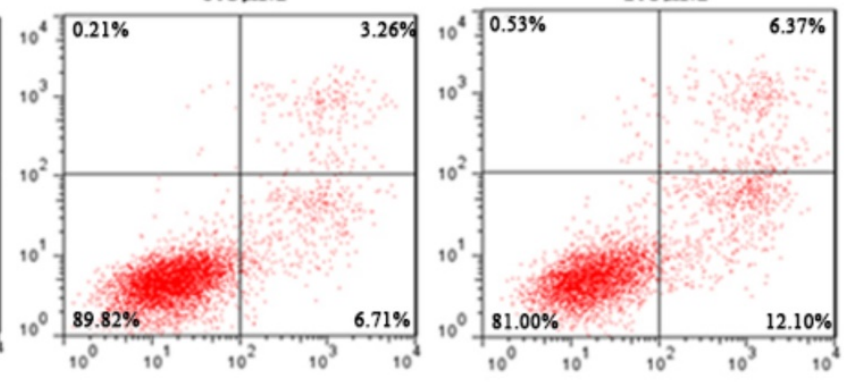

$\overline{2}$
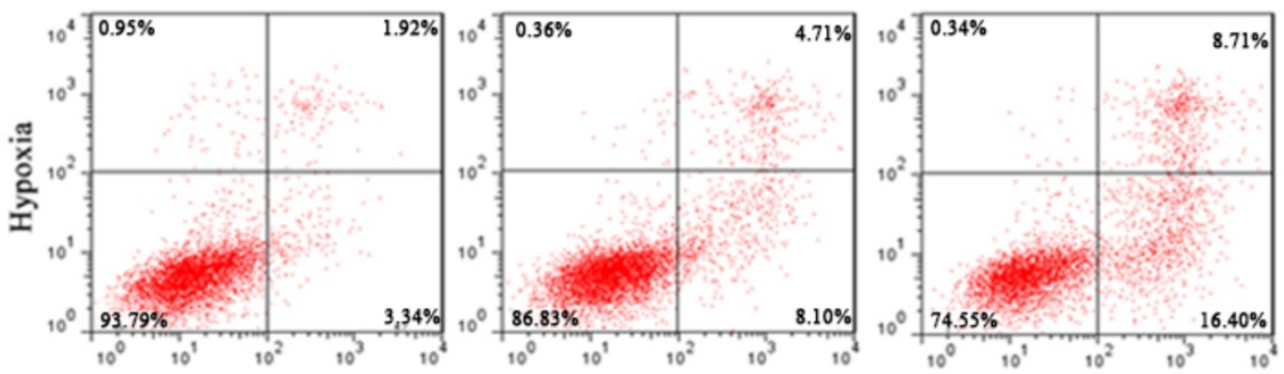

Annexin V- FITC

Figure 1. GNA inhibits proliferation and induces apoptosis in U266 cells. (A) The chemical structure of GNA. (B) The growth inhibitory effect of various concentrations of GNA on U266 cells for $24 \mathrm{~h}$ under normoxia and hypoxia was detected by CCK8 assay. Data are presented as mean \pm SD $(n=3)$. (C) The apoptosis rates of U266 cells after incubation with various concentrations of GNA for $24 \mathrm{~h}$ under normoxia and hypoxia were determined by flow cytometry. 


\section{Cytotoxicity assay}

Cytotoxic effect of GNA on proliferating cells was evaluated by CCK8 (Dojindo, Kumamoto, Japan). Gambogenic acid (Anhui University of Traditional Chinese Medicine, China) was dissolved in DMSO (Sigma-Aldrich), stored at $-20^{\circ} \mathrm{C}$, and diluted with RPMI-1640 when used in the experiment. U266 cells were seeded in 96-well plates at a density of $3 \times 10^{4}$ cells/well in $0.1 \mathrm{~mL}$ and incubated with serial dilutions of GNA. Plates were then cultured in a hypoxic culture, with normoxic culture as control. After 24 hours, the CCK-8 solution $(10 \mu \mathrm{L})$ was added to each well and incubated for additional 2 hours in the dark. The optical density (OD) was measured at $450 \mathrm{~nm}$ by Multiskan MK3 (Thermo Scientific, Shanghai, China). Then, cell viability was calculated as a percentage of viable cells in the GNA-treated group versus the untreated control.

\section{Flow cytometry}

Assessment of apoptotic cells were performed with Annexin V-FITC Apoptosis Detection Kit (Key-GEN, Nanjing, China). After being washed with ice-cold PBS twice, U266 cells were suspended with binding buffer and stained with (Annexin-V-FITC and PI) kit solution in dark for $15 \mathrm{~min}$. Thereafter, the apoptosis rate was determined by FACSCalibur Flow Cytometry (BD, USA).

\section{miRNA transfection}

U266 cells $\left(8 \times 10^{5}\right.$ cells) were seeded in 6-well plates. miR-21 inhibitor and miR-inhibitor negative control (NC) (GenePharma, Shanghai, China) was diluted in serum-free RPMI-1640 medium and mixed with Lipofectamin 2000 (GenePharma, Shanghai, China) for transfection following the manufacturer's instruction. The transfected cells were seeded into 6-well plates and cultured in serum-free RPMI-1640 medium at $37^{\circ} \mathrm{C}$ in a $\mathrm{CO}_{2}$ incubator.

\section{Quantitative real-time PCR assay}

Total RNA was extracted from cells by using RNAiso Plus (TaKaRa, Dalian, China) following the manufacturer's instructions. RNA was then reverse transcribed to cDNA with Mir-X miRNA First-Strand Synthesis kit (TaKaRa). Real-time PCR was carried out using Mir-X miRNA q-RT PCR kit (TaKaRa). The real-time PCR reaction contained: $9 \mu \mathrm{L}$ of $\mathrm{ddH}_{2} \mathrm{O}, 12.5$ $\mu \mathrm{L}$ of SYBR Advantage Premix (2X), $0.5 \mu \mathrm{L}$ of ROX Dye (50X), $0.5 \mu \mathrm{L}$ of miR21-specific primer $(10 \mu \mathrm{M})$, $0.5 \mu \mathrm{L}$ of mRQ $3^{\prime}$ primer $(10 \mu \mathrm{M}), 2.0 \mu \mathrm{L}$ of cDNA template. The program was $95^{\circ} \mathrm{C}$ for $10 \mathrm{~s}$, followed by 40 cycles of $95^{\circ} \mathrm{C}$ for $5 \mathrm{~s}, 60^{\circ} \mathrm{C}$ for $20 \mathrm{~s}$. The relative expression level of miR21 was normalized to that of internal control U6 using the comparative threshold cycle (Ct) method ( $\Delta \Delta \mathrm{Ct}$ method). The miR21-specific primer sequence was $5^{\prime}$-TAGCTTATCAGACTGATG TTGA-3'. The mRQ 3' primer, U6 Forward primer and U6 Reverse primer were provided by q-RT PCR kit (TaKaRa).

\section{Western blot analysis}

U266 cells of each group were collected and washed in lysis buffer. Total protein lysates from cells was extracted and western blot analyses were performed as described previously [18]. Blots were incubated respectively with different primary monoclonal antibodies (Santa Cruz Biotechnology, Santa Cruz, CA, USA) at $4^{\circ} \mathrm{C}$ overnight and incubated subsequently with corresponding horseradish peroxidase conjugated goat anti-rabbit secondary antibody (Santa Cruz Biotechnology) for 2 hours at room temperature. Enhanced chemiluminescence (ECL system; Amersham, UK) were used to visualize protein bands and Image J software (NIH, USA) were employed to quantify densitometry.

\section{Tumor xenograft model}

All the studies were conducted in adherence with protocols approved by the animal ethics committee of the Medical School, Southeast University. Six-week-old BALB/c nude mice (18-22 g) were purchased from the Shanghai National Center for Laboratory Animals. All mice were fed with sterile food in a specific pathogen-free facility. After injected subcutaneously with U266 cells $\left(1 \times 10^{7}\right.$ cells in 100ul of serum free RPMI-1640), the length (a) and width (b) of the tumor were measured every other day. When tumor volumes $\left(\mathrm{v} / \mathrm{mm}^{3}\right)$, calculated using the formula: $\mathrm{v}=\mathrm{ab}^{2} / 2$, reached approximately $50 \mathrm{~mm}^{3}$, the mice ( $n=5 /$ group) were randomly divided into two groups: saline water as the control group and the GNA group $(5 \mathrm{mg} / \mathrm{kg})$. The intravenous treatment was done every other day for seven times.

\section{Histopathologic examination}

After two weeks, all mice were sacrificed. Tumors and main organs were quickly removed and immediately immersed in $4 \%$ paraformaldehyde, dehydrated through a graded series of alcohol, and then embedded in paraffin wax blocks. 4- $\mu$ m-thick tissue sections of each group were prepared and then stained with hematoxylin-eosin. After staining, tissue sections were observed by microscope.

\section{Immunohistochemistry analysis}

Immunohistochemical staining with UltraSensitive S-P IHC Kit (Maixin, Fuzhou, China) were used to detect the expressions of Ki67 and PTEN. Tumor tissue sections were incubated with anti-Ki67 or anti-PTEN (1:100, Santa Cruz 
Biotechnology) at $4^{\circ} \mathrm{C}$ overnight. Then they were stained by a streptavidin-peroxidase system. Diaminobenzidine substrate was used to visualize the signal and counterstaining was done with hematoxylin.

\section{Statistical analysis}

Data were expressed as mean \pm standard deviation (SD) from at least 3 experiments performed in a parallel manner. Statistical analyses were performed using SPSS software (version 22.0; SPSS Inc, Chicago, IL, USA). Statistical significance of differences was determined by one-way analysis of variance among multiple groups. $\mathrm{P}<0.05$ was accepted as statistically significant.

\section{Results}

\section{GNA inhibits proliferation and induces apoptosis in $\mathbf{U} 266$ cells}

To determine whether GNA possessed antitumor properties under normoxic and hypoxic conditions, we evaluated its effect on the proliferation of U266 cells using CCK8 assay. Cells were treated with different concentrations of GNA under normoxia or hypoxia for 24 hours. The results showed that exposure to GNA resulted in a dose-dependent inhibition of cell proliferation (Figure 1B). Notably, the inhibitory effect was great even under hypoxic conditions. Similar to the antiproliferative effect, treatment of U266 cells with GNA induced apoptosis in a dose-dependent fashion and apoptotic activity was also greater under hypoxic conditions (Figure 1C).

\section{GNA attenuates hypoxia-induced upregulation of miR-2 1 expression}

Hypoxia is a remarkable feature of $\mathrm{MM}$ microenvironment and has activity of miR induction. To clarify associations between hypoxia, miR-21 and PTEN, we detected the expression levels of these cellular mediators in hypoxic U266 cells. As shown in Figure 2A and 2B, hypoxia upregulated the expression of miR-21 and miR-21 induced by hypoxia was associated with concurrent reductions in its target PTEN. We next examined the effect of GNA on miR-21 expression under normoxia and hypoxia. As illustrated in Figure 2C, GNA treatment decreased miR-21 expression compared to the group under hypoxia much more significantly than under normoxia. As miR-21 is a negative regulator of PTEN, the PTEN expression showed an opposite trend after GNA treatment (Figure 2D).

B

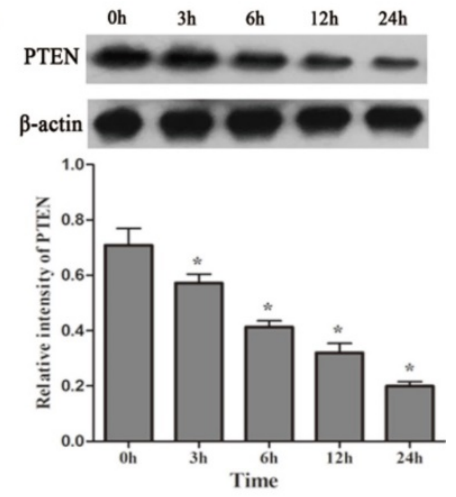

C

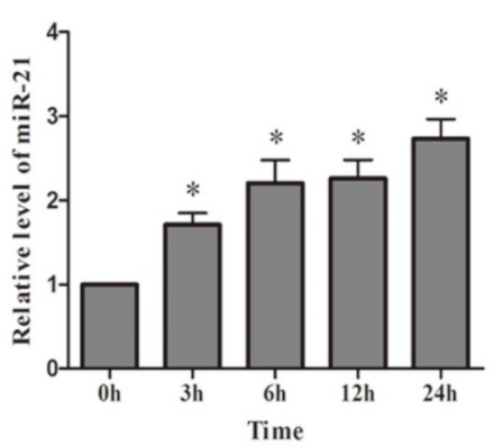

D

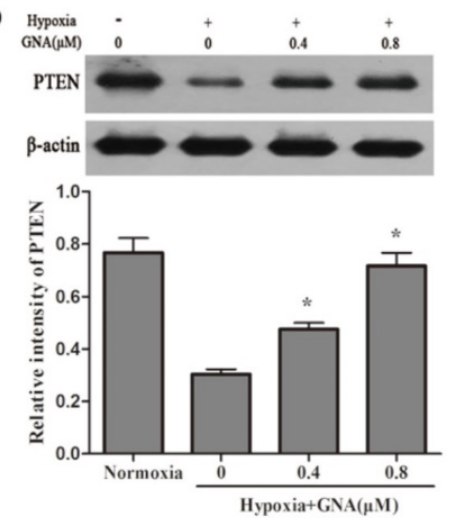

Figure 2. GNA attenuates hypoxia-induced upregulation of miR-21 levels. U266 cells were incubated under hypoxia for indicated different time, the expression of miR-21 (A) and PTEN protein levels $(B)$ were detected by qRT-PCR and western blot analysis, respectively. Data are presented as mean \pm SD $(n=3)$. $* \mathrm{P}<0.05$ compared to normoxia group. The miR-21 (C) and PTEN expression levels (D) in U266 cells treated with various concentrations of GNA under hypoxia were detected by $\mathrm{qRT}-\mathrm{PCR}$ and western blot analysis, respectively. Data are presented as mean $\pm \mathrm{SD}(\mathrm{n}=3)$. \#P<0.05 compared to normoxia alone group. $* \mathrm{P}<0.05$ compared to hypoxia alone group. 

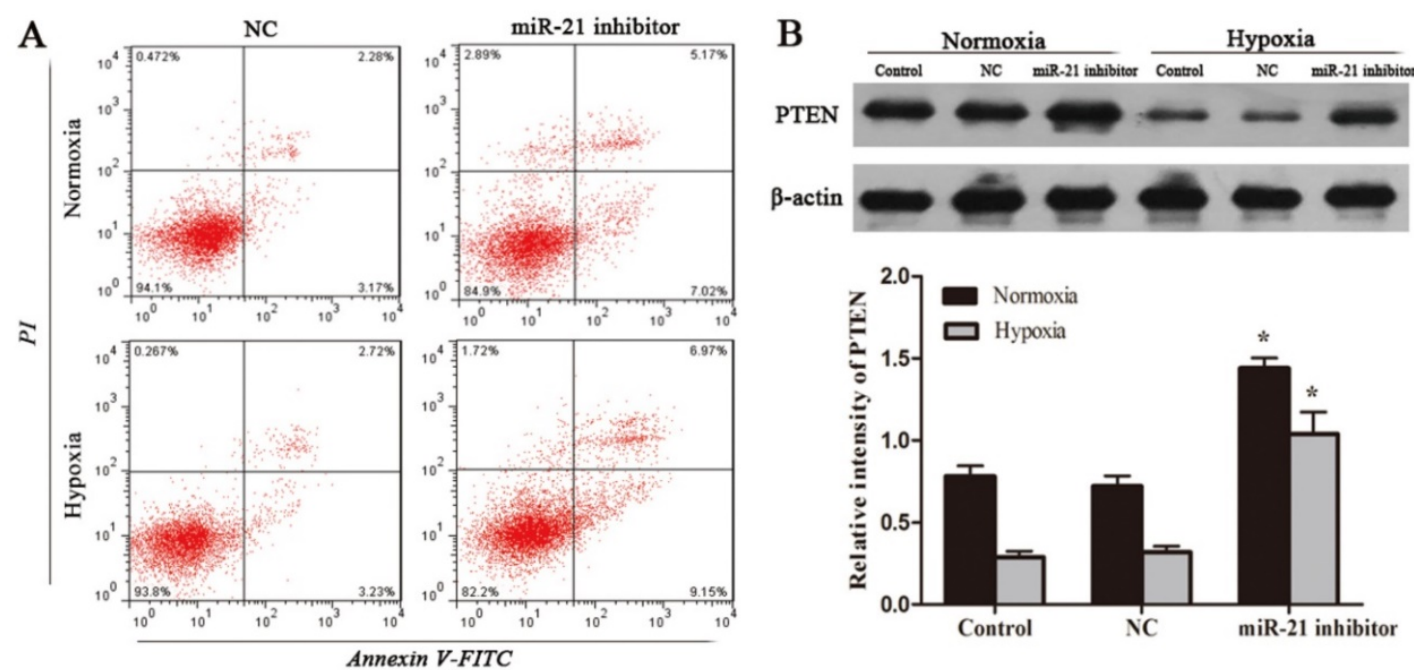

Figure 3. miR-21 inhibitor induces apoptosis and regulates PTEN expression in U266 cells. After transfection, cells were then cultured under normoxia or hypoxia for another 24 hours. (A) Apoptosis rates in each group were examined using flow cytometry. (B) The PTEN protein levels in U266 cells, were detected by the western blot analysis. Data are presented as mean $\pm \mathrm{SD}(\mathrm{n}=3)$. $* \mathrm{P}<0.05$ compared to control group.

\section{Inhibition of miR-21 induces apoptosis in U266 cells}

To verify if decreased miR-21 mediates cell apoptotic activity of GNA, U266 cells were transfected with either miR-21 inhibitor or miR-21 NC. After transfection, cells were then cultured under normoxia or hypoxia for another 24 hours. Apoptosis rates of cells were examined using flow cytometry. The results showed that compared with miR-21 NC groups, cells no matter under normoxia or hypoxia transfected with miR-21 inhibitor had significantly increased early and late apoptotic rates (Figure 3A). Western blot was used to evaluate the PTEN expression of each group. The results obtained indicated that miR-21 knowdown increased PTEN expression in normoxic U266 cells and attenuated reductions in PTEN protein levels under hypoxia (Figure 3B). This can explain that apoptosis rates were significantly raised by miR-21 inhibitor treatment.

\section{Inhibitory effect of GNA on HIF-1 $\alpha$ accumulation and STAT3 phosphorylation, which regulates miR-21 expression in hypoxic U266 cells}

HIF-1a and STAT3, key regulators in hypoxia adaptation, have been reported to be involved in hypoxia-induced miR-21. To evaluate whether GNA suppresses miR-21 expression involves in the repressors of HIF-1a or STAT3 signaling, we examined the expression levels of HIF-1a or p-STAT3 in response to GNA treatment under hypoxia. As shown in Figure 4A, hypoxia induces HIF-1a accumulation and STAT3 phosphorylation. In the presence of GNA, expression levels of HIF-1a and
p-STAT3 were significantly reduced in a concentration-dependent manner, while total STAT3 expression had no significant changes.

To substantiate the notion that HIF-1a or p-STAT3 acts as upstream mediators of miR-21 in the in vitro hypoxia model, we administered specific inhibitor against HIF-1a (2-ME) or p-STAT3 (S3I-201) prior to exposure to hypoxia and detected miR-21 expressions in hypoxic U266 cells. The results showed that blocking HIF-1a accumulation or STAT3 phosphorylation could in turn reduce miR-21 expression levels (Figure 4B). Western blot showed that PTEN expression levels were significantly increased in response to 2-ME and S3I-201 treatment (Figure 4C, 4D).

\section{GNA inhibits tumor growth and regulates expression levels of miR-2 1 and PTEN}

Nude mice that bore subcutaneous U266 xenografts were used to evaluate the tumor inhibitory effect of GNA (5 mg/ kg per 2 days) in vivo. After two-week intravenous treatment, the tumors were dissected and photographed (Figure 5A). Relative to the control group, the average tumor volumes of the GNA-treated group were significantly decreased $\left(950.5 \pm 189.3 \mathrm{~mm}^{3}\right.$ vs $359 \pm 74.8 \mathrm{~mm}^{3}, \mathrm{P}<0.05$, Figure $5 B)$. As shown in Figure 5C, the average tumor weight of the GNA-treated group was much lighter than the control group, indicating that GNA had a dramatic inhibitory effect on the growth of subcutaneous tumors. All mice were alive and no obvious side effects were noted throughout the study. GNA treatment had very minor effects on the body weight of mice (Figure 5D). Besides, no apparent histopathologic changes were observed in the main 
organs, including lung, heart, liver, kidney, and spleen (Figure 6A). The results demonstrated that GNA had minimal toxic effects for mice.

To further address whether the treatment mechanism agreed with our findings in vitro, qRT-PCR and immunohistochemistry were performed to detect the expression of miR-21, PTEN and ki67 in the xenograft specimens. As expected, the expression level of miR-21 were significantly decreased in xenograft tissue of GNA-treated group (Figure 6B). Meanwhile, PTEN, target of miR-21, was increased after GNA treatment. Finally, a pronounced decrease in the expression of ki67, the marker of tumor proliferation, was noted in GNA-treated group (Figure 6C). These data are consistent with the tumor growth curve, suggesting that GNA could attenuate tumor growth.

\section{Discussion}

MM represents the second most frequent hematologic disease with an annual incidence of 6.3 new cases per 100,000 individuals [19]. It remains incurable in many cases despite the variety of therapies already introduced, thus illustrating in-depth study of its pathogenesis and the urgent need for innovative therapeutic methods [20-22]. Traditional Chinese medicines have proven to be an attractive option for treatment of tumors due to their tumor suppressive activity. As a promising anti-tumor agent with multiple targets, GNA mediates a wide variety of functional anti-tumor effects [13-15]. In our previous study, we found GNA was markedly cytotoxic to normoxic MM cells. Herein, the results obtained from this study showed that GNA exhibits remarkable apoptotic activity against hypoxic MM cells and would encourage the further research for understanding the mechanism.
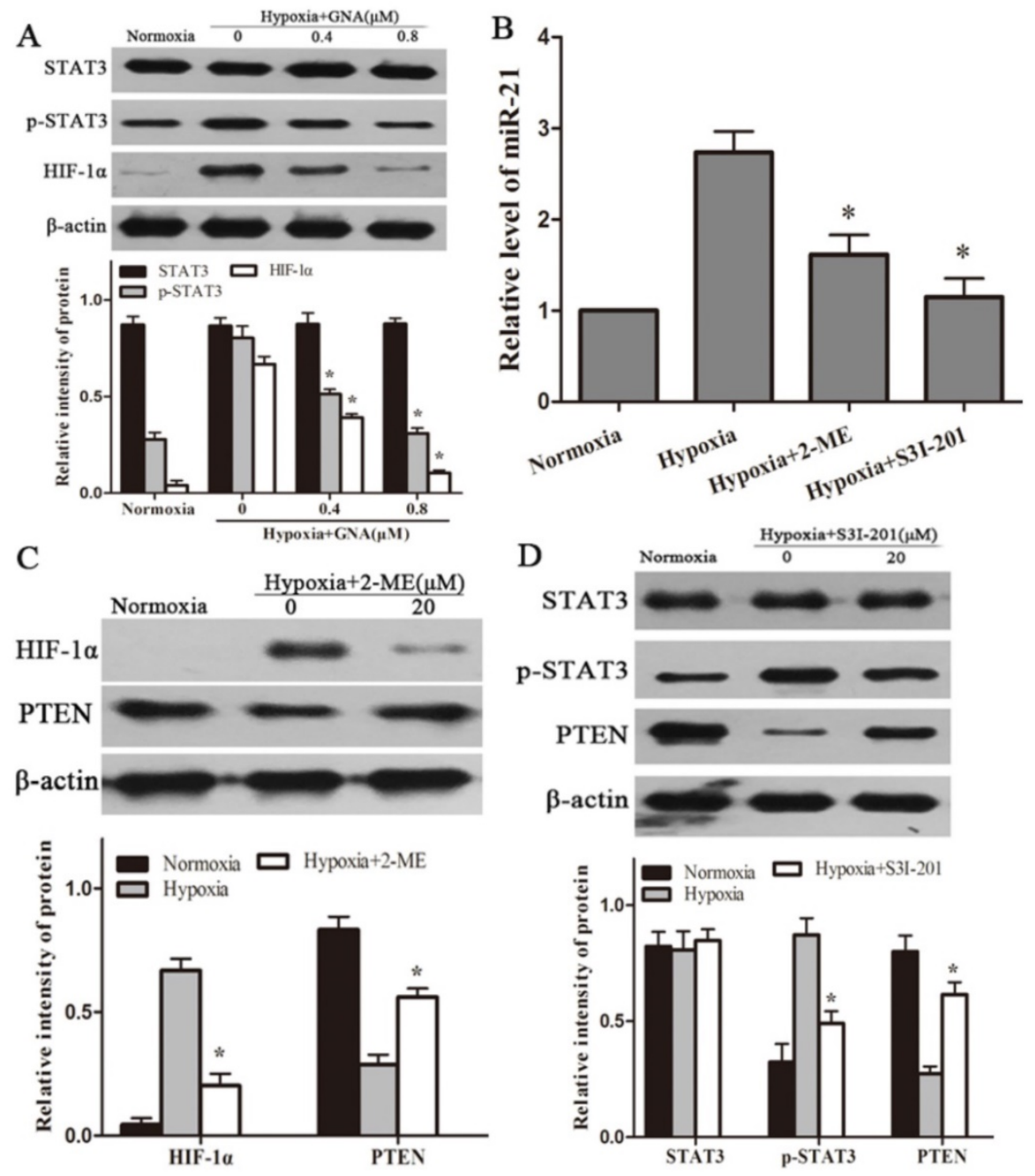

Figure 4. GNA regulates the expression of miR-21 via inhibition of HIF-1 $\alpha$ accumulation and STAT3 phosphorylation. (A) U266 cells were exposed to hypoxia in the presence or absence of GNA for $24 \mathrm{~h}$. The expressions of HIF-1 $\alpha$, STAT3, p-STAT3 were analyzed by western blots. Data are presented as mean $\pm \mathrm{SD}(\mathrm{n}=3)$. *P $<0.05$ compared to hypoxia alone group. U266 cells were exposed to hypoxia in the presence or absence of (2-ME) or (S3I-201) for 24 h. (B) The levels of miR-21 were detected by qRT-PCR. (C) 2-ME reduced the HIF-1 a protein expression and increased the PTEN protein expression. (D) S3I-201 reduced the $\mathrm{P}-\mathrm{STAT} 3$ protein expression and increased the PTEN protein expression. Data are presented as mean \pm SD $(n=3)$. *P $<0.05$ compared to hypoxia alone group. 
A

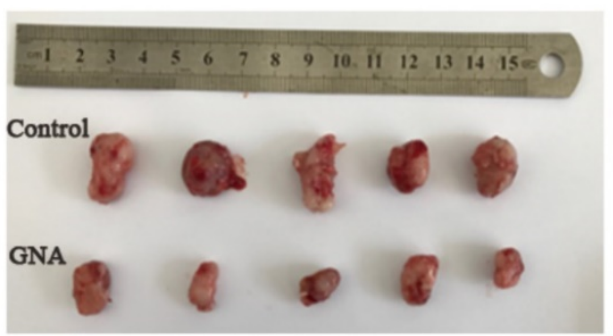

C

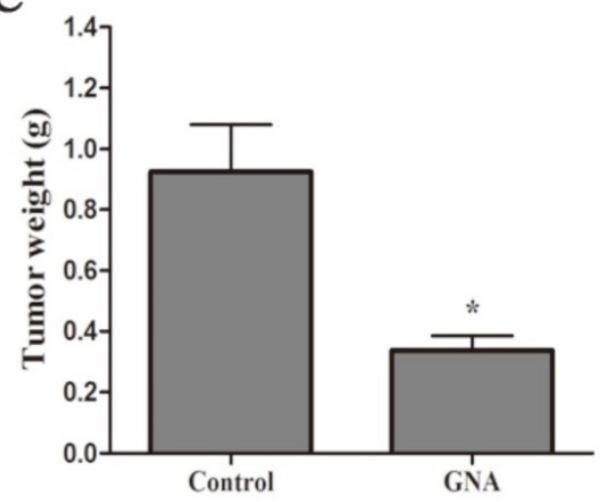

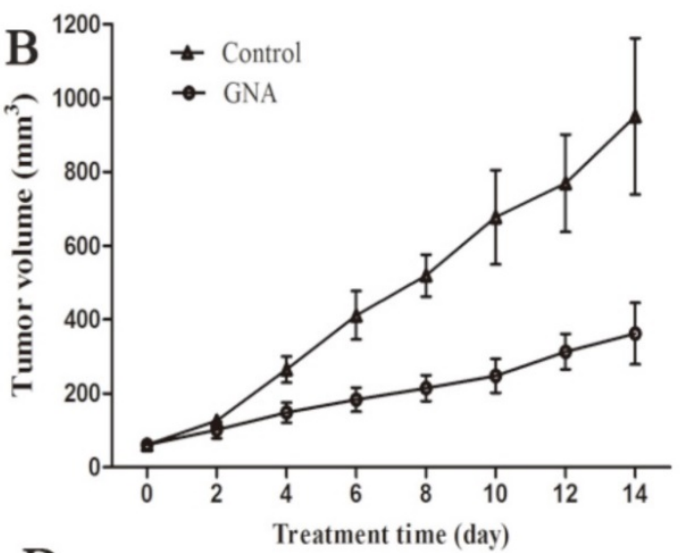

D

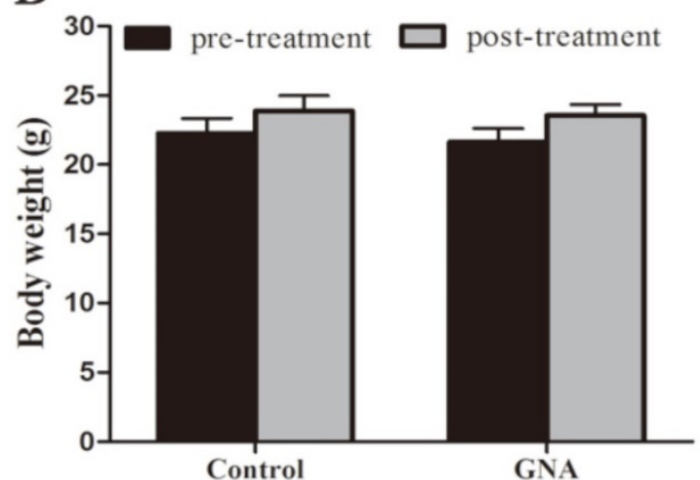

Figure 5. GNA inhibited tumor growth in U266 xenografts mice model. The BALB/c nude mice injected with U266 cells for a few days were treated with solvent or GNA ( $5 \mathrm{mg} / \mathrm{kg}$ ) every other day for 14 days. Then, the mice were sacrificed, tumor removed and photographed (A). The tumor size (B), tumor weight (C) and body weight $(D)$ were measured. Data are presented as mean $\pm S D(n=5) . * P<0.05$ compared to untreated controls.

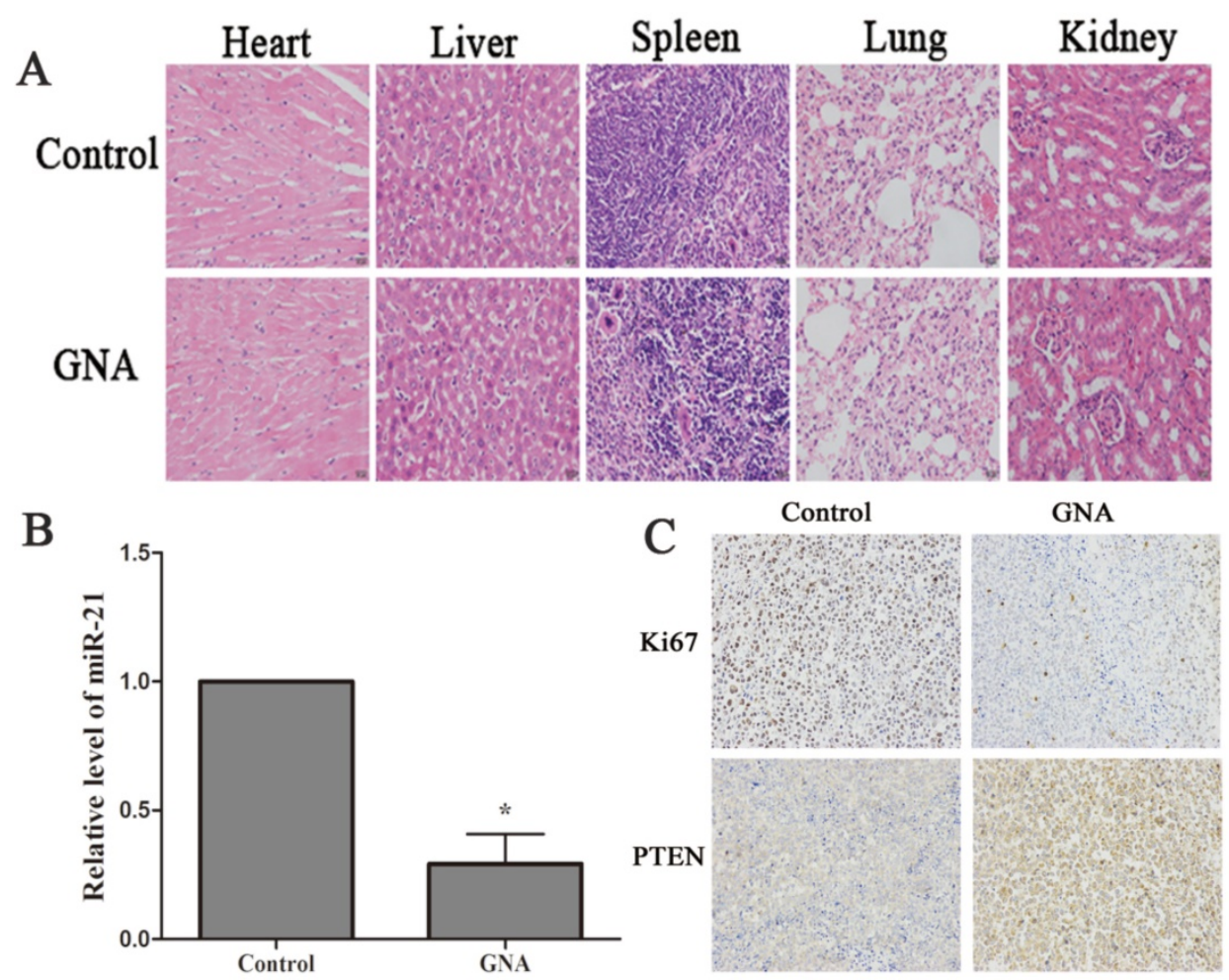

Figure 6. GNA inhibited tumor proliferation and regulates expression levels of miR-21 and PTEN. (A) Histopathological examination of organs in U266 xenograft model after the treatment (hematoxylin-eosin staining, $\times 200$ ). (B) miR-21 expressions in the tumor tissues were detected by $q R T$-PCR analysis. Data are presented as mean $\pm S D(n=5)$. $* P<0.05$ compared to untreated controls. (C) Immunohistochemistry was performed in tumor sections with antibodies of ki-67 and PTEN (immunohistochemistry, $\times 200)$. 
Hypoxia, which plays an important role in maintaining tumor microenvironment, accounts for aggressive tumor behavior and poor prognosis [23, 24]. Asosingh et al showed that the hypoxic BM environment has a role in the establishment of MM [25]. Work by Muz et al demonstrated that hypoxia induced MM cell dedifferentiation and increased drug resistance to proteasome inhibitors [26]. The underlined mechanisms accounted for the hypoxia-induced tumor malignancy progression have not been fully identified, but increasing findings have highlighted a relevant role for miRs in the regulation of hypoxic adaptation. miR-21 has been reported over-expressed in MM patients and associated with the occurrence and development of MM [10, 11, 27]. Direct studies have shown that there is a functional link between hypoxia and miR-21 expression in multiple tumors $[8,28]$. In the current study, we found that hypoxia increased miR-21 expression in U266 cells and miR-21 induced by hypoxia was associated with concurrent reductions in its target PTEN. After treatment with GNA, miR-21 expression in hypoxic U266 cells was strikingly downregulated in a dose-dependent manner. Consistent with prior reports, we found that downregulation of miR-21 expression triggered upregulation of suppressor gene PTEN, thereby decreasing multiple myeloma cell proliferation and survival [10, 29, 30].

Emerging evidence indicates that many miRs are transcriptional targets of HIF-1a and prevent hypoxic cells from apoptosis by targeting important downstream genes $[9,31]$. Here in our study, we demonstrated that inhibition of HIF-1a using its inhibitor 2-ME suppressed the expression of miR-21 under hypoxia and in turn increased PTEN expression. In addition to HIF-1a pathways, miRs also act as critical mediators of hypoxia signaling through other signaling pathways. Previous studies confirmed that activation of STAT3 regulates miR-21 expression, as there is a conserved STAT3-binding site locted in miR-21 genomic region [32]. Inhibition of the activation of STAT3 with the STAT3 inhibitor S3I-201 reduced the miR-21 expression, suggesting that p-STAT3 might work together with HIF-1a to induce the expression of miR-21 under hypoxia. The signaling proteins were further examined in the presence or absence of GNA under hypoxia. The results indicated that regulation of miR-21/PTEN by GNA under hypoxia is related with inhibition of HIF-1a accumulation and STAT3 phosphorylation.

Besides, tumor xenograft model was generated to evaluate the anti-tumor activity of GNA in vivo. As expected, intravenous administration of GNA significantly reduced the tumor size according to the tumor growth curve. Immune staining with Ki-67, a reliable means of rapidly estimating the growth fraction of neoplastic cell populations, further confirmed the anti-proliferative activity of GNA in U266 cells. qRT-PCR analysis and immune staining of the tumor sections revealed decrease of miR-21 expression and increase of PTEN expression, which was consistent with the findings in vitro.

In corollary, we conclude that GNA exerts antitumor activity in hypoxic MM cells by regulation of miR-21 via reducing HIF-1a accumulation and STAT3 phosphorylation. All our results provide evidences that GNA can affect malignant properties of U266 cells even under hypoxia both in vitro and in vivo. GNA appears to be a new potent therapeutic agent against human MM.

\section{Acknowledgments}

This work was supported by the National Natural Science Foundation of China (Grant No. 81370673), and the Key Medical Subjects of Jiangsu Province (Grant No. BL2014078), and the Key Discipline of Jiangsu Province (2016-2020).

\section{Competing Interests}

The authors have declared that no competing interest exists.

\section{References}

1. Avigan D, Rosenblatt J. Current treatment for multiple myeloma. N Engl J Med. 2014; 371: 961-2.

2. Kumar SK, Dispenzieri A, Lacy MQ, Gertz MA, Buadi FK, Pandey S, et al. Continued improvement in survival in multiple myeloma: changes in early mortality and outcomes in older patients. Leukemia. 2014; 28: 1122-8.

3. Munshi NC, Anderson KC. New strategies in the treatment of multiple myeloma. Clin Cancer Res. 2013; 19: 3337-44.

4. Ikeda S, Kitadate A, Abe F, Saitoh H, Michishita Y, Hatano Y, et al. Hypoxia-inducible microRNA-210 regulates the DIMT1-IRF4 oncogenic axis in multiple myeloma. Cancer Sci. 2017; 108: 641-52.

5. Mattick JS, Makunin IV. Non-coding RNA. Hum Mol Genet. 2006; 15:17-29.

6. Fabbri M, Garzon R, Andreeff M, Kantarjian HM, Garcia-Manero G, Calin GA. MicroRNAs and noncoding RNAs in hematological malignancies: molecular, clinical and therapeutic implications. Leukemia. 2008; 22: 1095-105.

7. Wang X, Li C, Ju S, Wang Y, Wang H, Zhong R. Myeloma cell adhesion to bone marrow stromal cells confers drug resistance by microRNA-21 up-regulation. Leuk Lymphoma. 2011; 52: 1991-8.

8. Kulshreshtha R, Ferracin M, Negrini M, Calin GA, Davuluri RV, Ivan M. Regulation of microRNA expression: the hypoxic component. Cell Cycle. 2007; 6: 1426-31.

9. Shen G, Li X, Jia YF, Piazza GA, Xi Y. Hypoxia-regulated microRNAs in human cancer. Acta Pharmacol Sin. 2013; 34: 336-41.

10. Leone E, Morelli E, Di Martino MT, Amodio N, Foresta U, Gulla A, et al. Targeting miR-21 inhibits in vitro and in vivo multiple myeloma cell growth. Clin Cancer Res. 2013; 19: 2096-106.

11. Wang JH, Zhou WW, Liu BX, Man DL, Yang ZD, Liu FR, et al. Expression and significance of miR-21 in multiple myeloma patients. Genet Mol Res. 2016; 15.

12. Song JZ, Yip YK, Han QB, Qiao CF, Xu HX. Rapid determination of polyprenylated xanthones in gamboge resin of Garcinia hanburyi by HPLC. J Sep Sci. 2007; 30: 304-9.

13. He Y, Ding J, Lin Y, Li J, Shi Y, Wang J, et al. Gambogenic acid alters chemosensitivity of breast cancer cells to Adriamycin. BMC Complement Altern Med. 2015; 15: 181.

14. Zhou J, Luo Y-H, Wang J-R, Lu B-B, Wang K-M, Tian Y. Gambogenic Acid Induction of Apoptosis in a Breast Cancer Cell Line. Asian Pacific Journal of Cancer Prevention. 2013; 14: 7601-5.

15. Mei W, Dong C, Hui C, Bin L, Fenggen $Y$, Jinging S, et al. Gambogenic acid kills lung cancer cells through aberrant autophagy. PLoS One. 2014; 9: e83604.

16. Yu X, Zhao Q, Zhang H, Fan C, Zhang X, Xie Q, et al. Gambogenic acid inhibits LPS-simulated inflammatory response by suppressing NF-kappaB and MAPK in macrophages. Acta Biochim Biophys Sin (Shanghai). 2016; 48: 454-61. 
17. Chen R, Zhang H, Liu P, Wu X, Chen B. Gambogenic acid synergistically potentiates bortezomib-induced apoptosis in multiple myeloma. J Cancer. 2017; 8: 839-51.

18. Liu P, Zhang H, Wu X, Guo L, Wang F, Xia G, et al. Tf-PEG-PLL-PLGA nanoparticles enhanced chemosensitivity for hypoxia-responsive tumor cells. Onco Targets Ther. 2016; 9: 5049-59.

19. Manier S, Powers JT, Sacco A, Glavey SV, Huynh D, Reagan MR, et al. The LIN28B/let-7 axis is a novel therapeutic pathway in multiple myeloma. Leukemia. 2016.

20. Lei W, Wang S, Yang C, Huang X, Chen Z, He W, et al. Combined expression of miR-34a and Smac mediated by oncolytic vaccinia virus synergistically promote anti-tumor effects in Multiple Myeloma. Sci Rep. 2016; 6: 32174.

21. Raninga PV, Di Trapani G, Vuckovic S, Tonissen KF. Targeted knockdown of DJ-1 induces multiple myeloma cell death via KLF6 upregulation. Apoptosis. 2016; 21: 1422-37.

22. Hu AX, Huang $\mathrm{ZY}$, Zhang L, Shen J. Potential prognostic long non-coding RNA identification and their validation in predicting survival of patients with multiple myeloma. Tumour Biol. 2017; 39: 1010428317694563.

23. Wang F, Zhang W, Guo L, Bao W, Jin N, Liu R, et al. Gambogic acid suppresses hypoxia-induced hypoxia-inducible factor-1alpha/vascular endothelial growth factor expression via inhibiting phosphatidylinositol 3-kinase/Akt/mammalian target protein of rapamycin pathway in multiple myeloma cells. Cancer Sci. 2014; 105: 1063-70.

24. Martin SK, Diamond P, Gronthos S, Peet DJ, Zannettino AC. The emerging role of hypoxia, HIF-1 and HIF-2 in multiple myeloma. Leukemia. 2011; 25: 1533-42.

25. Asosingh K, De Raeve H, de Ridder M, Storme GA, Willems A, Van Riet I, et al. Role of the hypoxic bone marrow microenvironment in 5T2MM murine myeloma tumor progression. Haematologica. 2005; 90: 810-7.

26. Muz B, de la Puente P, Azab F, Luderer M, Azab AK. Hypoxia promotes stem cell-like phenotype in multiple myeloma cells. Blood Cancer J. 2014; 4: e262.

27. Ma J, Liu S, Wang Y. MicroRNA-21 and multiple myeloma: small molecule and big function. Med Oncol. 2014; 31: 94.

28. Mace TA, Collins AL, Wojcik SE, Croce CM, Lesinski GB, Bloomston M. Hypoxia induces the overexpression of microRNA-21 in pancreatic cancer cells. J Surg Res. 2013; 184: 855-60.

29. Ou H, Li Y, Kang M. Activation of miR-21 by STAT3 induces proliferation and suppresses apoptosis in nasopharyngeal carcinoma by targeting PTEN gene. PLoS One. 2014; 9: e109929.

30. Wang S, Cheng Z, Yang X, Deng K, Cao Y, Chen H, et al. Effect of wild type PTEN gene on proliferation and invasion of multiple myeloma. Int J Hematol. 2010; 92: 83-94.

31. Zhou C, Tan W, Lv H, Gao F, Sun J. Hypoxia-inducible microRNA-488 regulates apoptosis by targeting Bim in osteosarcoma. Cellular Oncology. 2016; 39: 463-71.

32. Pratheeshkumar P, Son YO, Divya SP, Wang L, Zhang Z, Shi X. Oncogenic transformation of human lung bronchial epithelial cells induced by arsenic involves ROS-dependent activation of STAT3-miR-21-PDCD4 mechanism. Sci Rep. 2016; 6: 37227. 